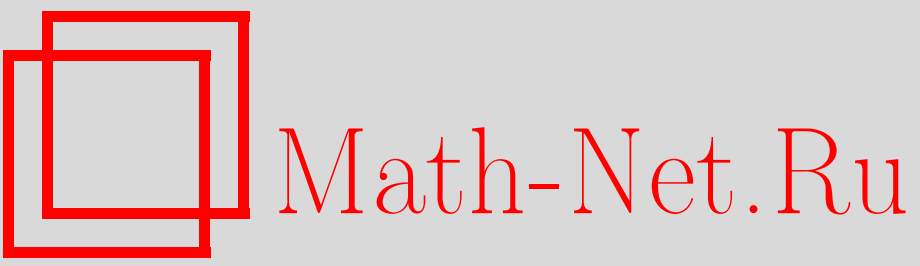

С. М. Сергеев, Вспомогательные трансфер-матрицы для трехмерных интегрируемых моделей, ТМФ, 2000, том 124, номер 3, 391-409

DOI: https://doi.org/10.4213/tmf646

Использование Общероссийского математического портала Math-Net.Ru подразумевает, что вы прочитали и согласны с пользовательским соглашением

http://www.mathnet.ru/rus/agreement

Параметры загрузки:

IP: 54.196.121.252

26 апреля 2023 г., 15:11:44 
ТЕОРЕТИЧЕСКАЯ

И МАТЕМАТИЧЕСКАЯ

ФИЗИКА

Том 124, № 3

сентябрь, 2000

(C) 2000 г.

С. М. Сергеев*

\section{ВСПОМОГАТЕЛЬНЫЕ ТРАНСФЕР-МАТРИЦЫ ДЛЯ ТРЕХМЕРНЫХ ИНТЕГРИРУЕМЫХ МОДЕЛЕЙ}

Формулируется локальный комбинаторный метод построения полного набора операторов, коммутирующих с трансфер-матрицей модели Замолодчикова-БажановаБакстера в вершинной формулировке. Для производящего функционала этого полного набора дается компактное функциональное уравнение.

\section{1. ВВЕДЕНИЕ}

Вот уже почти двадшать лет как была сформулирована первая нетривиальная трехмерная интегрируемая модель статистической механики - модель Замолодчикова. Позднее Бажанов и Бакстер получили ее обобщение на произвольное число спиновых состояний [1-3]. Бакстер вычислил статсумму модели [3, 4].

Вычислением статсуммы, однако, далеко не исчерпьвается изучение интегрируемых моделей. При рассмотрении любой дискретной квантовой интегрируемой модели всегда важно построение всего набора трансфер-матриц. В этой работе излагается метод построения полного (и даже переполненного) набора матрищ, коммутируюших с трансфер-матрицей модели Замолодчикова-Бажанова-Бакстера в вершинной формулировке [5]. В этом методе каждый интеграл движения сопоставляется с множеством путей определенного класса гомотопии на вспомогательной квадратной решетке с тороидальными граничными условиями.

Полный набор интегралов движения удобно описывать с помошью производяшего функционала, зависящего от двух спектральных параметров $\mathbf{J}(A, B)$. Такой функционал играет роль вспомогательной трансфер-матрицы. Функционал $\mathbf{J}$, однако, не имеет структуры следа вспомогательной матришы монодромии, а является чем-то более сложным. В методе построения этого функционала суммирование по путям похоже на построение некоторой статсуммы, а то, что суммируются матрицы, напоминает наличие спиновых цепочек в двумерных моделях.

Для нахождения спектра интегралов движения предлагается некоторое уравнение $N$-й степени ( $N$ - чис ло спиновых состояний модели) на функционал $\mathbf{J}$, занимаюшее место функциональных уравнений для обычных трансфер-матриц в двумерных моделях.

* Объединенный институт ядерных исследований, Дубна, Россия.

E-mail: sergeev_ms@mx.ihep.su 
В данной работе указан рецепт построения вспомогательной трансфер-матрицы $\mathbf{J}$ и найдено функциональное уравнение на нее. Эти результаты получаются с помощњю весьма простого метода квантовых вспомогательных линейных уравнений [6]. Поскольку этот метод относительно нов и потому малоизвестен, а его изложение занимает много места, мы приведем наши результаты без вывода в виде формулировок, определений и теорем, а вместо доказательства представим только лиш схемы доказательств в рамках общеизвестного квантового метода обратной задачи.

Далее мы договоримся об обозначениях, дав вершинную формулировку модели Замолодчикова-Бажанова-Бакстера и определив квантовую трансфер-матрицу Т этой модели. Затем будут сформулированы локальные комбинаторные правила построения вспомогательной трансфер-матрицы $\mathbf{J}(A, B)$ - производящего функционала для полного набора интегралов движения. В следуюшем разделе мы исследуем структуру J, вычислив некоторые необходимые в дальнейшем простейшие сохраняюшиеся операторы. После этого будет дана схема доказательства коммутативности матрищ $\mathbf{J}$ и $\mathbf{T}$ в терминах квантового метода обратной задачи. Далее мы выпишем функциональное уравнение на Ј. При этом будут рассмотрены и наиболее простые случаи: случай, когда число спиновых состояний равно двум (собственно модель Замолодчикова), и суперинтегрируемый случай $\left(\operatorname{tg}^{2}(\theta / 2)=-1\right)$. Доказательство функционального уравнения будет дано схематически в рамках уравнений вложенного бете-анзаца.

\section{2. БОЛЬЦМАНОВСКИЕ ВЕСА ИСХОДНОЙ ТРЕХМЕРНОЙ МОДЕЛИ}

В данной работе мы будем использовать вершинную формулировку модели Замолодчикова-Бажанова-Бакстера с $N$ состояниями [5]. Основным объектом в вершинной формулировке является матрица больцмановских весов, ассоциированная с трехмерной вершиной. Спиновые переменные, принимаюшие значения в $Z_{N}$, ассоциируются с ребрами кубической решетки, построенной из трехмерных вершин.

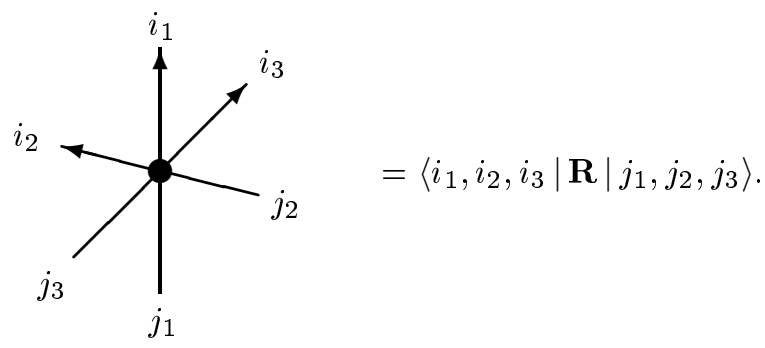

Рис. 1. Графическое представление матричных элементов $\mathbf{R}$.

На рис. 1 представлена трехмерная вершина, реберные спиновые переменные которой имеют значения $i_{1}, i_{2}, i_{3}$ и $j_{1}, j_{2}, j_{3}$. Наличие стрелок в вершине, различающих индексы $i_{\alpha}$ и $j_{\alpha}$, а также упорядочение $1,2,3$ существенно. Иными словами, все вершины считаются ориентированными. 
Для данных значений спиновых переменных больцмановский вес, соответствуюший вершине, обозначается как $R_{i_{1}, i_{2}, i_{3}}^{j_{1}, j_{2}, j_{3}}$. По виду больцмановские веса являются матричными элементами некоторой матрицы

$$
R_{i_{1}, i_{2}, i_{3}}^{j_{1}, j_{2}, j_{3}}=\left\langle i_{1}, i_{2}, i_{3}|\mathbf{R}| j_{1}, j_{2}, j_{3}\right\rangle
$$

где оператор $\mathbf{R}$ действует в упорядоченном тензорном произведении трех $N$-мерных линейных пространств, $\mathbf{R}: V_{N}^{\otimes 3} \mapsto V_{N}^{\otimes 3}$. Чтобы подчеркнуть упорядочение в тензорном произведении пространств $V$, а также то, что эти пространства имеют номера $1,2,3$, удобно использовать обозначение

$$
\mathbf{R}=\mathbf{R}_{1,2,3} .
$$

При рассмотрении тензорного произведения большего количества пространств считается, что оператор $\mathbf{R}_{1,2,3}$ дополнен произведением единичных матриц для компонент тензорного произведения с номерами, например, $4,5,6, \ldots$.

Как отмечалось, спиновые переменные принимают значения в $Z_{N}$, т.е. базис $|j\rangle$ пространства $V$ цикличен, $|j+N\rangle \equiv|j\rangle$.

Определим теперь вид матричных элементов R. Подробности содержатся в работе [5]. Пусть $p=(x, y)$ - точка на кривой Ферма

$$
x^{N}+y^{N}=1
$$

Здесь и далее всегда будет использоваться обозначение

$$
q=\exp \left(\frac{2 \pi i}{N}\right), \quad q^{N}=1
$$

Определим с точностью до нормировки функцию $W(p \mid a)$, первый аргумент которой точка на кривой Ферма, а второй аргумент принимает значение в $Z_{N}$, соотношением

$$
\frac{W(p \mid a)}{W(p \mid a-1)}=\frac{y}{1-q^{a} x} .
$$

С точностью до нормировки

$$
W(p \mid a)=W(p \mid 0) \prod_{\sigma=1}^{a} \frac{y}{1-q^{\sigma} x}
$$

Матричные элементы определяются формулой

$$
R_{i_{1}, i_{2}, i_{3}}^{j_{1}, j_{2}, j_{3}}=\delta_{i_{2}+i_{3}, j_{2}+j_{3}} q^{\left(j_{1}-i_{1}\right) j_{3}} \frac{W\left(p_{1} \mid i_{2}-i_{1}\right) W\left(p_{2} \mid j_{2}-j_{1}\right)}{W\left(p_{3} \mid j_{2}-i_{1}\right) W\left(p_{4} \mid i_{2}-j_{1}\right)}
$$

где всегда предполагается, что

$$
x_{1} x_{2}=q x_{3} x_{4}
$$


Независимыми в $\mathbf{R}$ являются только три точки на кривой Ферма, например $p_{1}, p_{2}, p_{3}$, поскольку $x_{4}$ можно найти из (8), а матрица весов инвариантна (с точностью до нормировки) относительно одновременного сдвига $y_{k} \mapsto q y_{k}, k=1,2,3,4$. Итак, получается, что матрица весов $\mathbf{R}$ является функцией от трех непрерывных и трех дискретных параметров.

Удобно выделить независимые параметры матрицы $\mathbf{R}$. Пусть

$$
\begin{aligned}
& \kappa_{1}=q^{\frac{1}{2}} \frac{x_{3}}{x_{1}}=q^{-\frac{1}{2}} \frac{x_{2}}{x_{4}}, \\
& \kappa_{2}=q^{\frac{1}{2}} \frac{x_{4}}{x_{1}}=q^{-\frac{1}{2}} \frac{x_{2}}{x_{3}}, \\
& \kappa_{3}=q^{\frac{1}{2}} \frac{y_{3} y_{4}}{y_{1} y_{2}}
\end{aligned}
$$

и

$$
u=\frac{y_{2}}{y_{3}}, \quad w=q^{-\frac{1}{2}} \frac{x_{1}}{y_{1}} \frac{y_{3}}{x_{3}}, \quad v=\frac{1}{q x_{3}} .
$$

Независимые параметры теперь - это значения $\kappa_{1}, \kappa_{2}, \kappa_{3}$ и фазы величин $u, v, w$. Следствием того, что величины $x_{k}$ и $y_{k}$ связаны соотношением Ферма, является система соотношений

$$
\frac{\left(1+w^{N}\right)\left(\kappa_{1}^{N}+v^{-N}\right)}{1+\kappa_{1}^{N}}=\frac{\left(1+\kappa_{1}^{N} v^{N}\right)\left(1+\kappa_{3}^{N} u^{N}\right)}{1+\kappa_{2}^{N}}=\frac{\left(1+w^{N}\right)\left(\kappa_{3}^{N}+u^{-N}\right)}{1+\kappa_{3}^{N}}=1
$$

Замечательное свойство матрицы $\mathbf{R}$ - ее геометрическая параметризация углами сферического треугольника, а именно

$$
\kappa_{1}^{N}=\left(\operatorname{tg} \frac{\theta_{1}}{2}\right)^{2}, \quad \kappa_{2}^{N}=\left(\operatorname{ctg} \frac{\theta_{2}}{2}\right)^{2}, \quad \kappa_{3}^{N}=\left(\operatorname{tg} \frac{\theta_{3}}{2}\right)^{2} .
$$

При этом $\theta_{\alpha}-$ двугранный угол между плоскостями, образующими линию с номером $\alpha$ в трехмерной вершине. Мы не будем пользоваться этой параметризацией (ее геометричность хороша только для параметризации уравнения тетраэдров), но отметим, что таким образом параметр $\kappa_{\alpha}$ ассоциирован с линией номера $\alpha$ и на самом деле является в определенном смысле спектральным параметром матрицы $\mathbf{R}$.

\section{3. СТАТИСТИЧЕСКАЯ МОДЕЛЬ НА КУБИЧЕСКОЙ РЕШЕТКЕ И ТРАНСФЕР-МАТРИЦА}

С помошью определенных выше больцмановских весов можно строить статистико-механические модели на трехмерных решетках. Простейшей решеткой является кубическая. Спиновые переменные ассоциируются с ребрами кубической решетки. Вершинам же присваиваются больцмановские веса $R$, зависящие от спинов окружающих вершину ребер. При этом спин каждого ребра относится к обеим вершинам, соединенным данным ребром. Для простоты решетка предполагается однородной, т.е. $R$ как функция своих остальных (не спиновых) параметров во всех вершинах является одной и той же. 


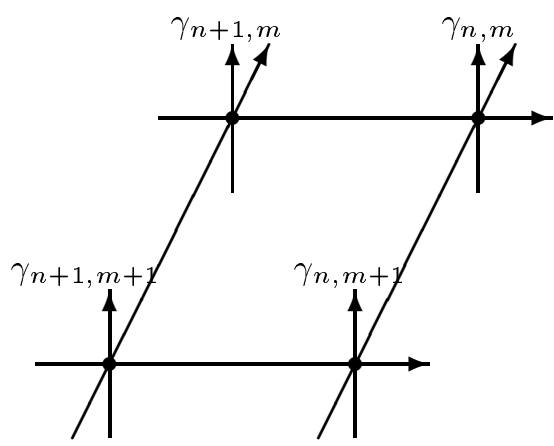

a

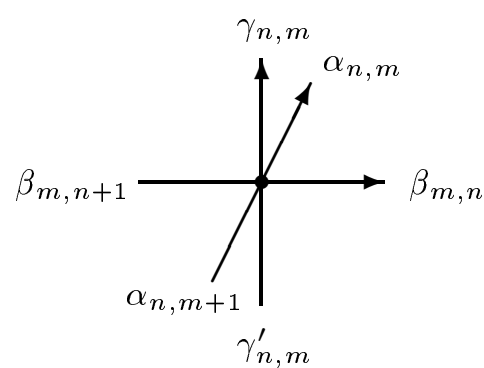

б

Рис. 2. Фрагмент трансфер-матрицы слой-слой.

Кубическая решетка может быть выбрана конечной и замкнутой, если наложить циклические граничные условия в трех направлениях решетки. Больцмановским весом всей решетки, соответствующим данной полной спиновой конфигурации, является произведение вершинных больцмановских весов. Сумма полного веса решетки по всем спиновым конфигурациям - это статистическая сумма модели.

Для вычисления статсуммы удобно определить трансфер-матрицу $\mathbf{T}$ типа слой-слой. Пусть $M_{1}$ и $M_{2}$-пространственные размеры кубической решетки в первом и втором направлениях согласно нумерации линий в $\mathbf{R}$-матрице. Рассмотрим сечение кубической решетки двумя близлежашими плоскостями, ортогональными линиям номер 3. Фрагмент такого сечения показан на рис. 2a. В этом сечении возникает набор открытых ребер третьего типа. Ребра же первого и второго типов замкнутые, и по их спинам надлежит выполнять суммирование. Статсумма, соответствуюшая такому сечению, является матрицей по спиновым переменным открытых ребер и записывается в виде

$$
\left\langle\gamma|\mathbf{T}| \gamma^{\prime}\right\rangle=\sum_{\{\alpha, \beta\}} \prod_{n, m}\left\langle\alpha_{n, m}, \beta_{m, n}, \gamma_{n, m}|\mathbf{R}| \alpha_{n, m+1}, \beta_{m, n+1}, \gamma_{n, m}^{\prime}\right\rangle
$$

Здесь по причине большого количества подобных линий мы использовали нумерацию $\alpha, \beta, \gamma$ вместо предыдущих обозначений $1,2,3$ и ввели целочисленные индексы для системы спиновых переменных, как это показано на рис. 2б. В частности, использовано мультииндексное обозначение

$$
\gamma=\left\{\gamma_{n, m}, n=1, \ldots, M_{2}, m=1, \ldots, M_{1}\right\}
$$

Циклические граничные условия означают, что

$$
\phi_{n+M_{2}, m}=\phi_{n, m+M_{1}}=\phi_{n, m} \quad \forall \phi \in\{\alpha, \beta, \gamma\} .
$$

Теперь статсумма кубической решетки, имеюшей $M_{3}$ слов в третьем направлении, есть

$$
\mathcal{Z}=\operatorname{Tr}\left(\mathbf{T}^{M_{3}}\right)
$$


Известно, что данная трехмерная модель интегрируема. Это следует из того, что матрицы Т образуют коммутативное семейство. В терминах четырех точек на кривой Ферма Т зависит (с точностью до нормировки) только от величин $x_{k}$ (три из которых независимы) и от фазы числа $y_{1} y_{2} / y_{3} y_{4}$. Вырождение параметрической зависимости происходит по причине инвариантости матрицы $\mathbf{T}$ относительно скрытых калибровочных преобразований $\mathbf{R}$ в первом и втором пространствах. В терминах параметров $\kappa_{1}$, $\kappa_{2}, \kappa_{3}$ и $u, v, w$ это означает зависимость от трех комплексных параметров $\kappa_{k}$ и от фазы числа $w$. Отметим, что изменение фазы $w$ соответствует всего лишь изменению нормировки матрищы $\mathbf{T}$ (что соответствует калибровочному преобразованию $\mathbf{R}$ в третьем пространстве), так что можно считать

$$
\mathbf{T}=\mathbf{T}\left(\kappa_{1}, \kappa_{2}, \kappa_{3}\right)
$$

В силу того что матрица $\mathbf{R}$ удовлетворяет трехмерному локальному условию интегрируемости - уравнению тетраэдров $[7,5]$, имеет место следующее соотношение:

$$
\mathbf{T}\left(\kappa_{1}, \kappa_{2}, \kappa_{3}\right) \mathbf{T}\left(\kappa_{4}, \kappa_{5}, \kappa_{3}\right)=\mathbf{T}\left(\kappa_{4}, \kappa_{5}, \kappa_{3}\right) \mathbf{T}\left(\kappa_{1}, \kappa_{2}, \kappa_{3}\right)
$$

т.е. две различные трансфер-матрицы коммутируют, если у них совпадает третий параметр (выделенность третьего параметра соответствует выделению третьего направления при построении трансфер-матрицы). С точки зрения квантового метода обратной задачи рассеяния такая коммутативность означает, что при разложении трансфер-матрицы $\mathbf{T}\left(\kappa_{1}, \kappa_{2}, \kappa_{3}\right)$ в ряд по некоторому базису функций от $\kappa_{1}$ и $\kappa_{2}$,

$$
\mathbf{T}\left(\kappa_{1}, \kappa_{2}, \kappa_{3}\right)=\sum_{a, b} f_{a}\left(\kappa_{1}\right) f_{b}\left(\kappa_{2}\right) \mathbf{t}_{a, b}\left(\kappa_{3}\right),
$$

мы получаем набор коммутативных матрищ $\mathbf{t}_{a, b}\left(\kappa_{3}\right)$, имеюших смысл полного набора интегралов движения динамической системы. Параметры $\kappa_{1}$ и $\kappa_{2}$ действительно являются спектральными параметрами модели, а параметр $\kappa_{3}$ в этом случае играет роль модуля.

Задача диагонализации трансфер-матрицы сводится к задаче диагонализации набоpa $\mathbf{t}_{a, b}\left(\kappa_{3}\right)$, в частности к получению его спектра. В данной работе дается альтернативньй метод (не используюший исходную квантовую трансфер-матрицу T) построения коммутативного семейства матриц $\mathbf{t}_{a, b}(\kappa)$, объединяемых в некоторую вспомогательную трансфер-матрицу $\mathbf{J}$. Для вспомогательной трансфер-матрицы $\mathbf{J}$ приводится функциональное соотношение, производяшее абелеву алгебру $\mathbf{t}_{a, b}$. Решения этого функционального соотношения дают спектр $\mathbf{t}_{a, b}$ и, таким образом, параметризуют собственные состояния исходной трансфер-матрицы $\mathbf{T}$.

\section{4. ВСПОМОГАТЕЛЬНАЯ КВАДРАТНАЯ РЕШЕТКА}

Рассмотрим вспомогательную квадратную решетку с циклическими граничными условиями, вершины $V$ которой пронумерованы парой индексов $V_{n, m}, \quad n \in Z_{M_{2}}$, 


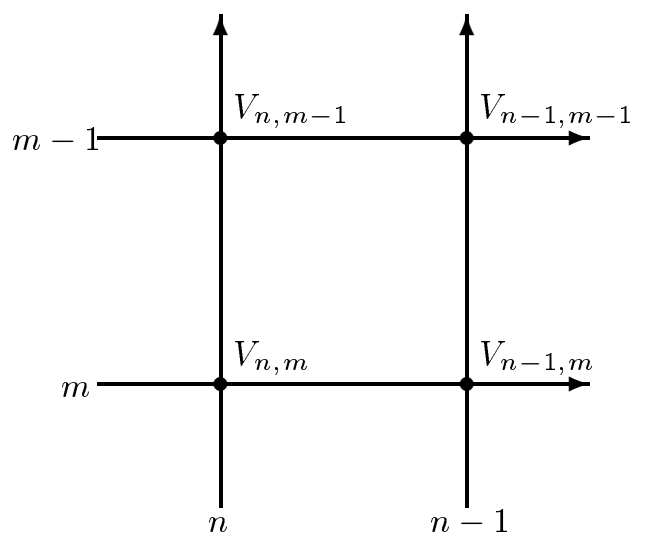

Рис. 3. Фрагмент вспомогательной решетки.

$m \in Z_{M_{1}}$, в точности так, как это было при построении трансфер-матрицы $\mathbf{T}$ (рис. 3). При этом индекс $n$ нумерует вертикальные линии, а индекс $m$ - горизонтальные.

Определим на этой решетке множество путей особого вида. Пусть каждый такой путь проходит следующим образом:

а) по каждой клетке решетки ровно один раз;

б) через каждую вершину решетки ровно один раз;

в) выходит из каждой вершины строго определенным способом, а именно вверх и влеBO.

Последнее требование означает, что у любого пути есть всего четыре возможности пройти сквозь вершину, и эти возможности изображены на рис. 4.

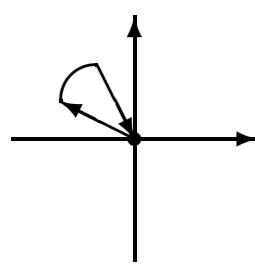

1

a

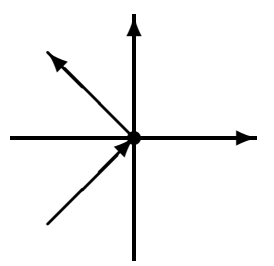

$\mathbf{w}$

б

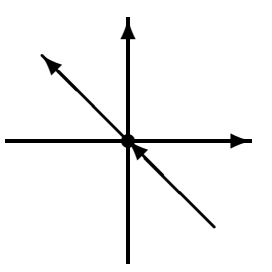

$\kappa \mathbf{u} \mathbf{w}$

B

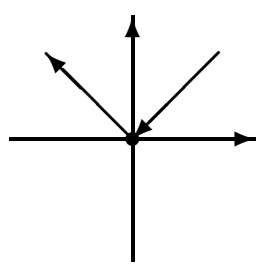

$q^{1 / 2} \mathbf{u}$

$\Gamma$

Рис. 4. Прохождение путей сквозь вершину.

Пример части некоторого пути приведен на рис. 5а. Естественно, каждый путь распадается на несколько связных компонент, и простейшая компонента связности есть тривиальная петля, как на рис. $4 \mathrm{a}$.

Вследствие циклических граничных условий вспомогательная решетка является тором, циклы которого обозначим как $\mathcal{A}$ - цикл обхода тора снизу вверх - и $\mathcal{B}$ - цикл обхода тора в направлении слева направо - в соответствии со стрелками вспомогательной решетки (см. рис. 5б). Все пути можно классифицировать по их гомотопическим классам. Гомотопическим классом несвязного пути является суперпозиция гомотопичеких 


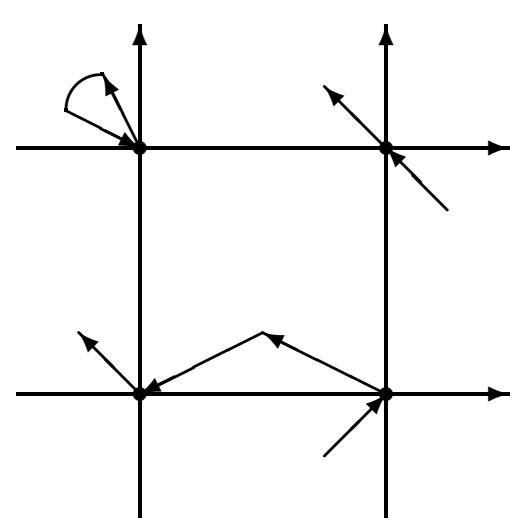

a

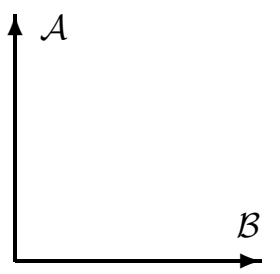

б

Рис. 5. Пример фрагмента пути и циклы.

классов компонент связности. В силу заданного направления выходов путей возможные гомотопические классы путей имеют вид

$$
\mathcal{W}(P)=a \mathcal{A}-b \mathcal{B}, \quad 0 \leqslant a \leqslant M_{2}, \quad 0 \leqslant b \leqslant M_{1},
$$

где $P$ - некоторьй путь, а $\mathcal{W}(P)$ - его гомотопический класс.

Далее ассоциируем с каждой вершиной вспомогательной решетки $N$-мерное линейное циклическое пространство $V$ ( $V$ уже использовалось в определении матрицы $\mathbf{R}$ в первом разделе). То, что для вершин и для пространств используется одна и та же буква, не приведет к неоднозначностям. Для каждого $V$ определим базисные циклические операторы $\mathbf{u}$ и $\mathbf{w}$ их действием на ортонормированный базис $V$ :

$$
\mathbf{u}|\gamma\rangle=q^{\gamma}|\gamma\rangle, \quad \mathbf{w}|\gamma\rangle=|\gamma+1\rangle
$$

Мы используем тот же самый базис $V$, что и при определении матричных элементов $\mathbf{R}$ в формуле (1). Очевидно, что

$$
\mathbf{u w}=q \mathbf{w} \mathbf{u}, \quad \mathbf{u}^{N}=\mathbf{w}^{N}=1 .
$$

Мы будем работать с тензорным произведением $M_{1} M_{2}$ копий пространства $V$, так что полное гильбертово пространство системы имеет базис

$$
|\gamma\rangle=\left|\gamma_{1,1}, \ldots, \gamma_{n, m}, \ldots\right\rangle
$$

Компоненты же тензорного произведения операторов будем обозначать парой индексов, например

$$
\mathbf{u}_{n, m}=1 \otimes 1 \otimes \cdots \otimes 1 \otimes \underbrace{\mathbf{u}}_{n, m \text {-е место }} \otimes 1 \otimes \cdots
$$

и т.д.

Ассоциируем далее с каждым прохождением пути сквозь вершину некоторую комбинацию операторов $\mathbf{u}$ и $\mathbf{w}$ для этой вершины, как это показано на рис. 4 . При этом на 
рис. 4в $\kappa$ - числовой параметр, общий для всех вершин. С некоторым полным путем $P$ мы ассоциируем произвдедение этих вершинных факторов, соответствующих всем вершинным фрагментам пути. Обозначим это произведение вершинных вкладов пути $P$ как $\mathbf{J}_{P}(\kappa)$, где подчеркивается наличие параметра $\kappa$ в списке вершинных вкладов. Вопросов с упорядочением не возникает, поскольку ассоциированные с разными вершинами операторнозначные множители коммутируют. Заметим, что всем тривиальным петлям соответствуют единичные множители в $\mathbf{J}_{P}(\kappa)$.

Выделим далее все пути $P$ с гомотопическим классом

$$
\mathcal{W}(P)=a \mathcal{A}-b \mathcal{B}
$$

Пусть

$$
\mathbf{J}_{a, b}(\kappa)=(-1)^{M_{1} M_{2}} \sum_{P: \mathcal{W}(P)=a \mathcal{A}-b \mathcal{B}}(-1)^{\sigma(P)} \mathbf{J}_{P}(\kappa)
$$

где $\sigma(P)$ - число петель в пути $P$, включая тривиальные. Описанные правила, при которых сначала вводится путь $P$ определенного оператора $\mathbf{J}_{P}$, устроенного как тензорное произведение коммутируюших операторов, а затем выполняется суммирование по всем возможным путям, весьма напоминают построение гамильтонианов для одномерных спиновых цепочек. Поэтому эти правила можно назвать “двумерной спиновой цепью".

Операторы $\mathbf{J}_{a, b}$ удобно объединить в операторнозначный полином

$$
\mathbf{J}(A, B \mid \kappa)=\sum_{a=0}^{M_{2}} \sum_{b=0}^{M_{1}} A^{a} B^{b} \mathbf{J}_{a, b}(\kappa)
$$

Заметим также, что операторы $\mathbf{J}_{a, b}(\kappa)$ между собой не коммутируют.

Теперь можно сформулировать следующую теорему.

ТЕОРема 1. Функционал $\mathbf{J}(A, B)$ сохраняется трансфер-матрицей $\mathbf{T}$,

$$
\mathbf{T}\left(\kappa_{1}, \kappa_{2}, \kappa_{3}\right) \mathbf{J}\left(A, B \mid \kappa_{3}\right)=\mathbf{J}\left(A, B \mid \kappa_{3}\right) \mathbf{T}\left(\kappa_{1}, \kappa_{2}, \kappa_{3}\right)
$$

Более того, система $\mathbf{J}_{a, b}(\kappa)$ содержит единожды переполненный набор интегралов движсения матрицы $\mathbf{T}$.

Доказательство теоремы 1 будет проведено следующим образом. Сначала мы проанализируем структуру полинома $\mathbf{J}(A, B)$ и объясним, что означает фраза "единожды переполненный набор интегралов", а затем (в разделе 6) изложим схему алгебраического доказательства этой теоремы. 


\section{5. АНАЛИЗ СИСТЕМЫ ОПЕРАТОРОВ $\mathbf{J}_{a, b}$}

УТВЕРЖДЕНИЕ 1. Операторы $\mathbf{J}_{a, b}$, определенные формулой (25), удовлетворяют коммутационным соотношениям

$$
\mathbf{J}_{a, b} \mathbf{J}_{a^{\prime}, b^{\prime}}=q^{b a^{\prime}-a b^{\prime}} \mathbf{J}_{a^{\prime}, b^{\prime}} \mathbf{J}_{a, b} .
$$

Заметим, что оператор $\mathbf{J}_{0,0}=1$. Он коммутирует со всеми остальными операторами. Доказательства этого утверждения мы пока не имеем.

Множество индексов $(a, b)$ операторов $\mathbf{J}_{a, b}, 0 \leqslant a \leqslant M_{2}, 0 \leqslant b \leqslant M_{1}$, образует целочисленный прямоугольник на плоскости $(a, b)$. Каков периметр этого прямоугольника?

Периметрические операторы $\mathbf{J}_{a, b}$ соответствуют минимальным или же максимальным намоткам путей на тор. И в том, и в другом случаях соответствуюшие $\mathbf{J}_{a, b}$ легко вычисляются комбинаторно.

Рассмотрим путь класса $\mathcal{A}$, проходящий через все вершины вертикальной линии с номером $n$. Во всех остальных вершинах при этом подразумеваются тривиальные петли. Этому пути соответствует (с точностью до знаков) выражение

$$
\mathbf{W}_{n}=\prod_{m=1}^{M_{1}} \mathbf{w}_{n, m} .
$$

С помощью таких выражений легко вычислить, рассматривая пути $P$ класса $\mathcal{W}(P)=$ $a \mathcal{A}$, сумму

$$
\sum_{a=0}^{M_{2}} A^{a} \mathbf{J}_{a, 0}=\prod_{n=1}^{M_{2}}\left(1-(-1)^{M_{1}} A \mathbf{W}_{n}\right)
$$

Аналогично для путей $P$ класса $\mathcal{W}(P)=-b \mathcal{B}$ введем величины

$$
\mathbf{U}_{m}=\prod_{n=1}^{M_{2}} \mathbf{u}_{n, m}
$$

и вычислим сумму

$$
\sum_{b=0}^{M_{1}} B^{b} \mathbf{J}_{0, b}=\prod_{m=1}^{M_{1}}\left(1-(-1)^{M_{2}} q^{\frac{M_{2}}{2}} B \mathbf{U}_{m}\right) .
$$

Несколько сложнее, но тоже достаточно просто вычисляются частичные производяшие функционалы путей

$$
P: \mathcal{W}(P)=a \mathcal{A}-M_{1} \mathcal{B}
$$

имеющие вид

$$
\sum_{a=0}^{M_{2}} A^{a} \mathbf{J}_{a, M_{1}}=(-1)^{M_{1}\left(M_{2}-1\right)} q^{\frac{M_{1} M_{2}}{2}} \prod_{m=1}^{M_{1}} \mathbf{U}_{m} \prod_{n=1}^{M_{2}}\left(1-(-1)^{M_{1}} q^{-\frac{M_{1}}{2}} \kappa^{M_{1}} A \mathbf{W}_{n}\right)
$$

Для путей

$$
P: \mathcal{W}(P)=M_{2} \mathcal{A}-b \mathcal{B}
$$


находим

$$
\sum_{b=0}^{M_{1}} B^{b} \mathbf{J}_{M_{2}, b}=(-1)^{M_{2}\left(M_{1}-1\right)} \prod_{m=1}^{M_{1}}\left(1-(-1)^{M_{2}} \kappa^{M_{2}} B \mathbf{U}_{m}\right) \prod_{n=1}^{M_{2}} \mathbf{W}_{n}
$$

Видно, что периметрическими элементами системы $\mathbf{J}_{a, b}$ являются симметричные полиномы от $\mathbf{U}_{m}$ и $\mathbf{W}_{n}$. Используя точный вид $\mathbf{R}$-матрищы (7), нетрудно убедиться, что не только симметрические полиномы, но и сами матрицы $\mathbf{U}_{m}, \mathbf{W}_{m}$ коммутируют с $\mathbf{T}$.

Итак, установлено, что периметрические элементы системы $\mathbf{J}_{a, b}$ соответствуют $M_{1}+$ $M_{2}$ независимым нетривиальным операторам: это $M_{1}$ штук матриц $\mathbf{U}_{m}$ и $M_{2}$ штук матриц $\mathbf{W}_{n}$. Имеется также единичная матрица $\mathbf{J}_{0,0}=1$. Алгебра матриц $\mathbf{U}_{m}$ и $\mathbf{W}_{n}$ определяется соотношениями

$$
\mathbf{U}_{m} \mathbf{W}_{n}=q \mathbf{W}_{n} \mathbf{U}_{m} \quad \forall n, m .
$$

Выделим произвольным способом из системы матриц $\mathbf{U}_{m}, \mathbf{W}_{n}$ некоммутативную пару $\mathbf{U}, \mathbf{W}$ (например, при простейшем выборе $\mathbf{U}=\mathbf{U}_{1}$ и $\mathbf{W}=\mathbf{W}_{1}$; выбор, приводящий к обобщенной киральной модели Поттся [8], есть

$$
\mathbf{U}^{M_{1}}=\prod_{m=1}^{M_{1}} \mathbf{U}_{m}
$$

и $\left.\mathbf{W}=\mathbf{W}_{1}\right)$ и отнормируем все остальные матрицы $\mathbf{U}_{m}, \mathbf{W}_{n}$,

$$
\mathbf{U}_{m}=\mathbf{U U}_{m}^{\prime}, \quad \mathbf{W}_{n}=\mathbf{W} \mathbf{W}_{n}^{\prime}
$$

Теперь система матриц $\mathbf{U}_{m}^{\prime}, \mathbf{W}_{n}^{\prime}$ стала коммутативной. Аналогично поступим со всеми элементами системы $\mathbf{J}_{a, b}$ :

$$
\mathbf{J}_{a, b}=\mathbf{U}^{b} \mathbf{W}^{a} \mathbf{t}_{a, b}
$$

В силу соотношений $(28)$ операторы $\mathbf{t}_{a, b}$ коммутируют между собой и с $\mathbf{U}, \mathbf{W}$. Это, кстати, означает, что матрицы

$$
\tau(A, B \mid \kappa)=\sum_{a, b} A^{a} B^{b} \mathbf{t}_{a, b}
$$

при одинаковых $\kappa$ образуют коммутирующее семейство.

УТВЕРЖДЕНИЕ 2. Набор $M_{1} M_{2}$ следующих коммутативных операторов:

а) $\left(M_{1}-1\right)\left(M_{2}-1\right)$ операторов $\mathbf{t}_{a, b}$, отвечающих внутренности прямоугольника $(a, b): 0<a<M_{2}$ u $0<b<M_{1}$;

б) $M_{1}-1$ независимых операторов $\mathbf{U}_{m}^{\prime}$;

в) $M_{2}-1$ независимых операторов $\mathbf{W}_{n}^{\prime}$;

г) любой оператор, являющийся функиией от $\mathbf{U}, \mathbf{W}$ и имеющий невырожсенный спектр, 
образует полный набор операторов для гильбертова пространства состояний $V^{\otimes M_{1} M_{2}}$.

Это утверждение о полноте, так же как и предыдушее, мы приводим без доказательства.

Таким образом, система интегралов движения полна, а из-за наличия некоммутативной пары переполнена. Очевидно, что операторы $\mathbf{U}, \mathbf{W}$ имеют смысл канонических переменных центра инерции.

\section{6. СХЕМА АЛГЕБРАИЧЕСКОГО ДОКАЗАТЕЛЬСТВА ТЕОРЕМЫ 1}

Схема алгебраического доказательства теоремы 1 основана на приемах квантового метода обратной задачи: двумерной $R$-матрице, $L$-операторе и уравнении сплетения.
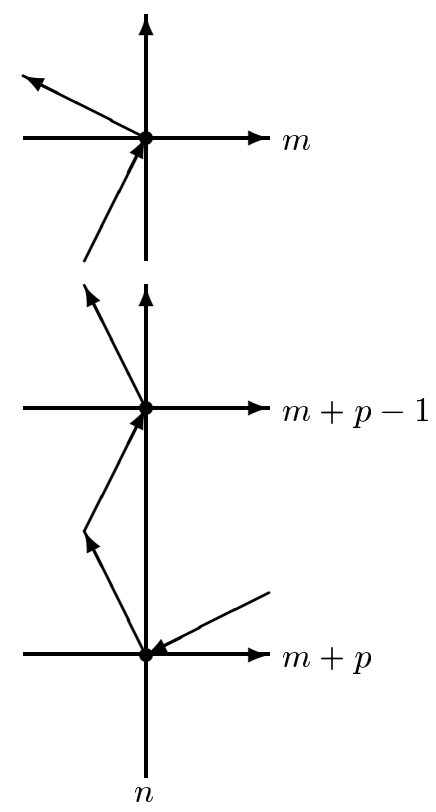

Рис. 6. Образование матричного элемента $L_{m, m+p}^{(1)}$.
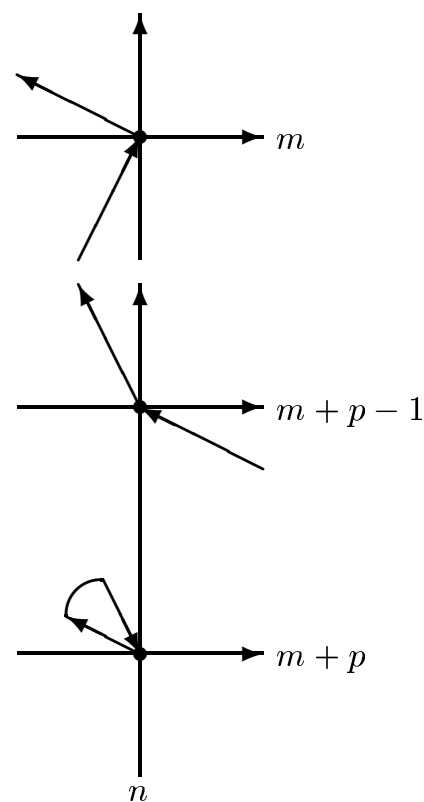

Факторизуем функционал $\mathbf{J}(A, B)$ по параметру $B$ :

$$
\mathbf{J}(A, B)=\sum_{b=0}^{M_{1}} B^{b} \mathbf{J}_{b}(A) .
$$

Коммутативность операторов $\mathbf{J}_{0}(A)$ и $\mathbf{J}_{M_{1}}(A)$ с $\mathbf{T}$ уже обсуждалась, она тривиальна. Вернемся на вспомогательную плоскость и рассмотрим теперь функционал $\mathbf{J}_{1}(A)$. Он образуется путями $P$ с гомотопическим классом $\mathcal{W}(P)=a \mathcal{A}-\mathcal{B}, 0 \leqslant a \leqslant M_{2}$. Выделим мысленно на вспомогательной плоскости $n$-ю вертикальную линию. Очевидно, что все пути обсуждаемого класса пересекают каждую вертикальную линию ровно один раз. Две возможности такого пересечения представлены на рис.6. Оба нарисованных 
фрагмента обладают одним и тем же свойством: они подходят к $n$-й вертикальной линии над горизонтальной линией с номером $m+p$ и перескакивают с $n$-й вертикальной линии на $(n+1)$-ю сразу над горизонтальной линией с номером $m$. Определим операторнозначную матрицу $L^{(1)}(A)_{m, m^{\prime}}$ размера $M_{1} \times M_{1}$ по следующим правилам:

$$
\begin{aligned}
\left(L^{(1)}(A)_{n}\right)_{m, m+p} & =-(-x)^{p}\left(\kappa \mathbf{u}_{n, m+p-1}-q^{\frac{1}{2}} \mathbf{u}_{n, m+p}\right) \mathbf{w}_{n, m} \ldots \mathbf{w}_{n, m+p-1}, \\
\left(L^{(1)}(A)_{n}\right)_{m, m} & =-\left(q^{\frac{1}{2}} \mathbf{u}_{n, m}-(-x)^{M_{1}} \kappa \mathbf{u}_{n, m-1} \mathbf{W}_{n}\right),
\end{aligned}
$$

где $x^{M_{1}} \equiv A$. Множитель $(-x)^{p}$ маркирует степень перескока с $(m+p)$-й линии на $m$-ю. В качестве аргумента матрицы $L$ мы указываем не $x$, а $A$, поскольку простым (но не единственным) диагональным калибровочным преобразованием все степени $x$, кроме $x^{M_{1}}$, можно почти везде устранить, нарушив, однако, при этом $M_{1}$-цикличность последовательности $m, m+1, \ldots, m+p, \ldots, m+M_{1} \sim m$. Матричные элементы $L^{(1)}$, определенные в формулах $(40),(41)$, очевидно, являются всевозможными фрагментами $\mathbf{J}_{a, 1}$, относяшимися к вертикальной линии $n$. Простые комбинаторные выкладки показывают, что

$$
-\mathbf{J}_{1}(A)=\operatorname{Tr}\left(L^{(1)}(A)_{1} L^{(1)}(A)_{2} \ldots L^{(1)}(A)_{n} \ldots L^{(1)}(A)_{M_{2}}\right),
$$

т.е. оператор $\mathbf{J}_{1}(A)$ является трансфер-матрицей от $L$-оператора $L^{(1)}$. Напомним обшепринятую терминологию: $M_{1}$-мерное пространство $L$-оператора, по которому берется след матришы монодромии, называется вспомогательным пространством, а $N^{M_{1}}$-мерное пространство, отвечающее $M_{1}$ вейлевским парам $\mathbf{u}_{n, m}, \mathbf{w}_{n, m}, m=1, \ldots, M_{1}$,- квантовым пространством. При этом трансфер-матрица $\mathbf{J}_{1}(A)$ называется вспомогательной трансфер-матрицей для квантовой трансфер-матрицы $\mathbf{T}$.

Несложно показать, что матрица $L^{(1)}(A)$ является $L$-оператором для векторного представления $\omega_{1}$ алгебры $U_{q}\left(\widehat{s l\left(M_{1}\right)}\right)$, т.е.

$$
R\left(\frac{A}{A^{\prime}}\right)_{1,2} L^{(1)}(q A)_{1, n} L^{(1)}\left(A^{\prime}\right)_{2, n}=L^{(1)}\left(q A^{\prime}\right)_{2, n} L^{(1)}(A)_{1, n} R\left(\frac{A}{A^{\prime}}\right)_{1,2},
$$

где $R_{1,2}$ - тригонометрическая $R$-матрица для векторного представления $U_{q}\left(\widehat{s l\left(M_{1}\right)}\right)$. В этом уравнении сплетения встречается лишний, несколько непривычный множитель $q$ в аргументах $L$-операторов. Это та цена, которая заплачена за трехмерную инвариантность модели Замолодчикова-Бажанова-Бакстера по сравнению с обобщенной киральной моделью Поттса $[8,2]$. Происхождение этого множителя связано с алгеброй $\mathbf{U}_{m}, \mathbf{W}_{n}$. Фактически это означает, что $L^{(1)}$ является $L$-оператором для $g l\left(M_{1}\right)$ и $\mathbf{W}_{n}$, $\mathbf{U}_{m}$ имеют смысл $U_{1}$-зарядов.

Коммутативность $\mathbf{J}_{1}(A)$ с $\mathbf{T}$ есть следствие соотношения сплетения $L$-операторов по квантовым пространствам. Выделим из квантовой трансфер-матришы $\mathbf{T}$ фрагмент, отвечающий $n$-й линии,

$$
\left\langle\beta, \gamma|\mathbf{S}| \beta^{\prime}, \gamma^{\prime}\right\rangle=\sum_{\{\alpha\}} \prod_{m=1}^{M_{1}}\left\langle\alpha_{m}, \beta_{m}, \gamma_{m}|\mathbf{R}| \alpha_{m+1}, \beta_{m}^{\prime}, \gamma_{m}^{\prime}\right\rangle .
$$


Очевидно, что

$$
\mathbf{T}=\operatorname{Tr}_{\beta}\left(\mathbf{S}_{\beta, \gamma_{1}} \cdots \mathbf{S}_{\beta, \gamma_{n}} \cdots \mathbf{S}_{\beta, \gamma_{M_{2}}}\right) .
$$

Теперь, восстанавливая параметры $\kappa$, можно доказать соотношение

$$
\tilde{L}\left(A, \kappa_{2}\right)_{1, \beta} L\left(A, \kappa_{3}\right)_{1, \gamma} \mathbf{S}\left(\kappa_{1}, \kappa_{2}, \kappa_{3}\right)_{\beta, \gamma}=\mathbf{S}\left(\kappa_{1}, \kappa_{2}, \kappa_{3}\right)_{\beta, \gamma} L\left(A, \kappa_{3}\right)_{1, \gamma} \tilde{L}\left(A, \kappa_{2}\right)_{1, \beta}
$$

В этом уравнении оператор $\tilde{L}$ устроен так же, как и оператор $L$. Единственное отличие состоит в том, что для $\tilde{L}$ (помимо другого $\kappa \mapsto \kappa_{2}$ ) параметры образуюших вейлевских алгебр $\mathbf{u}$ и w его квантового пространства $\beta$ неединичны, т.е.

$$
\mathbf{u}|\beta\rangle=q^{\beta}|\beta\rangle, \quad \mathbf{w}|\beta\rangle=u^{-1}|\beta+1\rangle,
$$

где параметр $u$ определен в (10). Напомним, что именно из соотношения (46) и выводилась изначально $R$-матрица $\mathbf{S}$ для обобшенной киральной модели Поттса [8].

Итак, коммутативность $\mathbf{J}_{1}(A)$ с $\mathbf{T}$ условно доказана. Вернемся назад, к путям на вспомогательной решетке, и рассмотрим пути $P$ класса $\mathcal{W}(P)=a \mathcal{A}-b \mathcal{B}$ для всех $0 \leqslant$ $a \leqslant M_{2}$ и для фиксированного $1 \leqslant b<M_{1}$. Аналогичный комбинаторный анализ пересечения некоторым путем данного класса вертикальной линии позволит ввести $L$-оператор $L^{(b)}(A)_{n}$ матричной размерности

$$
\left(\begin{array}{c}
M_{1} \\
b
\end{array}\right) \times\left(\begin{array}{c}
M_{1} \\
b
\end{array}\right),
$$

что соответствует размерности фундаментального представления $\omega_{b}$ алгебры $s l\left(M_{1}\right)$. Оказывается, что $L^{(b)}(A)$ будут сплетаться по вспомогательным пространствам тригонометрическими $R$-матрицами представлений $\omega_{b}$ (правда, в соотношениях сплетения придется компенсировать наличие $U_{1}$-зарядов множителями $\left.q^{b}\right)$. По квантовым же пространствам эти $L$-операторы будут сплетаться той же самой матрицей $\mathbf{S}_{\beta, \gamma}$. Из квантового соотношения сплетения следует, что операторы

$$
\mathbf{J}_{b}(A) \sim \operatorname{Tr}\left(L^{(b)}(A)_{1} \ldots L^{(b)}(A)_{n} \ldots L^{(b)}(A)_{M_{2}}\right)
$$

коммутируют с $\mathbf{T}$. Тем самым вся теорема 1 условно доказана.

Отметим еше раз, что мы получали функционал $\mathbf{J}$ отнюдь не этим алгебраическим методом, а совершенно другим, называемым методом квантовой вспомогательной линейной задачи. В этом методе полином $\mathbf{J}(A, B)$ возникает как детерминант матрицы операторнозначных коэффициентов системы линейных уравнений, и его сохранение матрицей Т есть следствие некоторой модификации теоремы о пропорциональности детерминантов матриц коэффициентов эквивалентных систем однородных линейных уравнений. Приведем вид вспомогательной системы линейных уравнений для трансфер-матрицы Т из работ [6]:

$$
\left\langle\varphi_{n, m}\right|+\left\langle\varphi_{n-1, m}\right| q^{\frac{1}{2}} \mathbf{u}_{n, m}+\left\langle\varphi_{n, m+1}\right| \mathbf{w}_{m, n}+\left\langle\varphi_{n-1, m+1}\right| \kappa \mathbf{u}_{n, m} \mathbf{w}_{n, m}=0,
$$

где подразумеваются граничные условия $\varphi_{n, m+M_{1}}=\varphi_{n, m} A$ и $\varphi_{n-M_{2}, m}=\varphi_{n, m} B$. 


\section{7. ФУНКЦИОНАЛЬНОЕ СООТНОШЕНИЕ}

В предыдуших разделах рассматривалась система путей на вспомогательной решетке и были сформулированы правила сопоставления каждому пути $P$ некоторого оператора $\mathbf{J}_{P}$ с последующим взятием "статсуммы" по путям. Изменим слегка правила сопоставления, заменив подписи к вариантам прохождения пути сквозь вершину рис. 4 следуюшими:

$$
1 \mapsto 1, \quad \mathbf{w} \mapsto \varepsilon_{N} \mathbf{w}^{N}, \quad \kappa \mathbf{u w} \mapsto \kappa^{N} \mathbf{u}^{N} \mathbf{w}^{N}, \quad q^{\frac{1}{2}} \mathbf{u} \mapsto-\varepsilon_{N} \mathbf{u}^{N},
$$

где

$$
\varepsilon_{N}=(-1)^{N-1} .
$$

При нашей нормировке для каждой вершины $\mathbf{u}^{N}=\mathbf{w}^{N}=1$ и новые правила сопоставляют пути $P$ число $J_{P}^{f}$. Числовая статсумма определяется аналогично операторнозначной с точностью до замены $A$ и $B$ на $A^{N}$ и $B^{N}$ :

$$
J_{f}\left(A^{N}, B^{N} \mid \kappa^{N}\right)=\sum_{a, b} A^{a N} B^{b N}(-1)^{M_{1} M_{2}} \sum_{P: \mathcal{W}(P)=a \mathcal{A}-b \mathcal{B}}(-1)^{\sigma(P)} J_{P}^{f} .
$$

В силу однородности вспомогательной решетки статсумма $J_{f}$ вычисляется просто, и мы имеем

$$
J_{f}\left(A^{N}, B^{N} \mid \kappa^{N}\right)=\prod_{\substack{x_{1}=A^{N} \\ y^{M_{2}}=B^{N}}}\left(1-\varepsilon_{N} y+\varepsilon_{N} x+\kappa^{N} x y\right),
$$

где произведение берется по $M_{1} M_{2}$ множителям, отвечающим различным корням $M_{1}$-й и $M_{2}$-й степеней для $x$ и $y$.

Теорема 2. Имеет место соотношение

$$
\underset{\mathbf{U}, \mathbf{W}}{\operatorname{det}} \mathbf{J}(A, B \mid \kappa)=J_{f}\left(A^{N}, B^{N} \mid \kappa^{N}\right)
$$

әде $\operatorname{det} \mathbf{U}, \mathbf{W}$ обозначает детерминант по блоку некоммутативной пары $\mathbf{U}, \mathbf{W}$ в блочном представлении оператора $\mathbf{J}$.

Очевидно, что соотношение (54) производит абелеву алгебру операторов $\mathbf{t}_{a, b}$.

Итак, функциональное соотношение (54) сформулировано, метод его доказательства мы обсудим в следуюшем разделе, а пока посмотрим на простейший по $N$ случай детерминанта (54).

В случае $N=2$ детерминант по паре $\mathbf{U}, \mathbf{W}$ вычисляется крайне просто:

$$
\underset{\mathbf{U}, \mathbf{W}}{\operatorname{det}} \mathbf{J}(A, B \mid \kappa)=\frac{1}{2}(\tau(A, B) \tau(-A,-B)+\tau(-A, B) \tau(A,-B)),
$$

где трансфер-матрица $\tau$ определена формулой (38), а из периметрических выражений для $\mathbf{J}$ легко получить граничные условия на $\tau$, просто заменив $\mathbf{U}_{m}$ на $\mathbf{U}_{m}^{\prime}$ и $\mathbf{W}_{n}$ на $\mathbf{W}_{n}^{\prime}$. 
В наиболее интересном секторе, когда $\mathbf{U}_{m}^{\prime}=\mathbf{W}_{n}^{\prime}=1$ (а это и есть обобщенная киральная модель Поттса) и для простоты полагается, что $M_{1}=M_{2}=M$, граничные условия для полинома $\tau$ имеют вид

$$
\begin{aligned}
\sum_{a} A^{a} \mathbf{t}_{a, 0} & =\left(1-(-1)^{M} A\right)^{M}, \\
\sum_{b} B^{b} \mathbf{t}_{0, b} & =\left(1-\left(-q^{\frac{1}{2}}\right)^{M} B\right)^{M}, \\
\sum_{a} A^{a} \mathbf{t}_{a, M} & =q^{\frac{M^{2}}{2}}\left(1-\left(-q^{-\frac{1}{2}} \kappa\right)^{M} A\right)^{M}, \\
\sum_{b} B^{b} \mathbf{t}_{M, b} & =\left(1-(-\kappa)^{M} B\right)^{M} .
\end{aligned}
$$

Можно еще упростить функциональное соотношение, рассмотрев так называемый суперинтегрируемый случай, когда $\kappa=q^{1 / 2}=i$. В этом случае

$$
\begin{aligned}
\tau(A, B)= & \left(1-(-1)^{M} A\right)^{M}\left(1-\left(-q^{\frac{1}{2}}\right)^{M} B\right)^{M}+\sum_{a, b=1}^{M-1} \mathbf{t}_{a, b}^{\prime} A^{a} B^{b} \\
& J_{f}\left(A^{2}, B^{2}\right)=\left(1-A^{2}\right)^{M}\left(1-(-1)^{M} B^{2}\right)^{M}
\end{aligned}
$$

Переопределив

$$
x=(-1)^{M} A, \quad y=\left(-q^{\frac{1}{2}}\right)^{M} B,
$$

получаем уравнение

$$
\tau(x, y) \tau(-x,-y)+\tau(-x, y) \tau(x,-y)=2\left(1-x^{2}\right)^{M}\left(1-y^{2}\right)^{M}
$$

с граничным условием $\tau(x, y)=(1-x)^{M}(1-y)^{M}+\tau^{\prime}$, где $\tau^{\prime}$ - полином по $x, y$ степеней от 1 до $M-1$.

\section{8. СХЕМА АЛГЕБРАИЧЕСКОГО ДОКАЗАТЕЛЬСТВА ТЕОРЕМЫ 2}

Вместо точного доказательства теоремы 2 мы опять ограничимся лишь схемой, по которой эту теорему можно в принципе доказать с помошью методов алгебраического бете-анзаца.

Сначала рассмотрим случай $M_{1}=2$ и посмотрим на структуру уравнения (54). Мы снова факторизуем коммутативные трансфер-матришы по параметру $B$, как это делалось в разделе о доказательстве теоремы 1:

$$
\tau(A, B)=\sum_{b=0}^{2} B^{b} \tau_{b}(A) .
$$

При этом скалярные матрицы $\tau_{0}(A)$ и $\tau_{2}(A)$ имеют вид

$$
\tau_{0}(A)=\prod_{n=1}^{M_{2}}\left(1-A \mathbf{W}_{n}^{\prime}\right), \quad \tau_{2}(A)=q^{M_{2}} \mathbf{U}_{1}^{\prime} \mathbf{U}_{2}^{\prime} \prod_{n=1}^{M_{2}}\left(1-q^{-1} \kappa^{2} A \mathbf{W}_{n}^{\prime}\right) .
$$


В этих терминах оператор $\mathbf{J}(A, B)$ представляется в виде

$$
\mathbf{J}(A, B)=B \mathbf{U}\left(B^{-1} \mathbf{U}^{-1} \tau_{0}(A \mathbf{W})+\tau_{1}(A \mathbf{W})+B \mathbf{U} \tau_{2}(A \mathbf{W})\right)
$$

Детерминант по блоку $\mathbf{U}, \mathbf{W}$, естественно, не зависит от выбора базиса в этом блоке. Удобно выбрать базис, в котором оператор $\mathbf{W}$ диагонален,

$$
\mathbf{U}|\alpha\rangle=|\alpha-1\rangle, \quad \mathbf{W}|\alpha\rangle=q^{\alpha}|\alpha\rangle
$$

При этом

$$
B^{-1} \mathbf{U}^{-1} \mathbf{J}(A, B)|\alpha\rangle=\tau_{0}\left(q^{\alpha} A\right) B^{-1}|\alpha+1\rangle+\tau_{1}\left(q^{\alpha} A\right)|\alpha\rangle+\tau_{2}\left(q^{\alpha} A\right) B|\alpha-1\rangle .
$$

Выберем далее $A$ и $B$ так, чтобы $J_{f}=0$, т.е. выберем пару значений $x$ и $y$, удовлетворяющих условиям $x^{2}=A^{N}, y^{M_{2}}=B^{N}$,

$$
1-\varepsilon_{N} y+\varepsilon_{N} x+\kappa^{N} x y=0 .
$$

Если вместо $x$ и $y$ ввести переменные $\lambda$ и $\eta$, заданные соотношениями $\lambda^{2}=A, \eta^{M_{2}}=$ $B$, то формула (66) перепишется в виде

$$
1-\varepsilon_{N} \eta^{N}+\varepsilon_{N} \lambda^{N}+\kappa^{N} \lambda^{N} \eta^{N}=0,
$$

что обычно называется бакстеровской кривой для киральной модели Поттса.

На поверхности $J_{f}=0$ уравнение $(54)$ означает, что существует вектор

$$
|Q\rangle=\sum_{\alpha} Q_{\alpha}|\alpha\rangle
$$

такой, что

$$
\mathbf{J}(A, B)|Q\rangle=0
$$

причем $Q_{\alpha}$ являются просто алгебраическими дополнениями матрицы $\mathbf{J}$ по любой строке в базисе $|\alpha\rangle$. Из структуры матричных элементов (65) очевидно, что

$$
Q_{\alpha}=Q\left(q^{\alpha} A\right)
$$

(зависимость от $B$ подразумевается). Соотношение (70) приводит к уравнению

$$
\tau_{1}(A) Q(A)=-\tau_{0}\left(q^{-1} A\right) B^{-1} Q\left(q^{-1} A\right)-\tau_{2}(q A) B Q(q A),
$$

где величины $A$ и $B$ задаются точкой на бакстеровской кривой. Уравнение (71) есть бакстеровское $(T-Q)$-уравнение алгебраического бете-анзаца для однородной киральной модели Поттса в случае неединичных $U_{1}$-зарядов $\mathbf{W}_{n}^{\prime}, \mathbf{U}_{m}^{\prime}$.

Заметим, что процедура получения $(T-Q)$-уравнения Бакстера из функционального уравнения (54) обратима: исходя из уравнения (71) и принимая во внимание произвольность выбора $x$ и $y$ с точностью до $M_{1}$-й и $M_{2}$-й степеней из единицы соответственно, мы 
получаем уравнение (54) с точностью до нормировки, которая затем легко фиксируется через граничные условия $\tau_{0}(A)$ и $\tau_{M_{1}}(A)$. Аналогичная, но технически гораздо более утомительная процедура позволяет вывести уравнение (54) для произвольного $M_{1}$ на основании более высокого аналога $(T-Q)$-уравнения $(71)$, известного как система уравнений вложенного бете-анзаца.

Итак, предложена схема доказательства теоремы 2 с помощью метода вложенного бете-анзаца. Уравнение (54) выводилось с помощью метода квантовой вспомогательной линейной задачи, где оно просто тривиально. Левая и правая части (54) суть детерминант одной и той же весьма большой блочной матрицы (размером $M_{1} M_{2} N^{M_{1} M_{2}} \times$ $M_{1} M_{2} N^{M_{1} M_{2}}$ ), рассчитанный в разных последовательностях блочного вложения.

\section{9. ЗАКЛЮЧЕНИЕ}

В данной работе сформулирован локальный, комбинаторный метод построения вспомогательной трансфер-матрицы $\mathbf{J}(A, B)$ для квантовой трансфер-матрицы $\mathbf{T}$ типа слойслой в вершинной формулировке однородной модели Замолодчикова-БажановаБакстера. Приведено также функциональное уравнение для вспомогательной трансфер-матрищы, решения которого определяют спектр модели.

В конструкции функционала $\mathbf{J}(A, B)$ и уравнении (54) использовались вершинные параметры $\mathbf{u}^{N}=1, \mathbf{w}^{N}=1$ и $\kappa$, одинаковые для всей вспомогательной решетки. В обшем же случае параметры $\mathbf{u}^{N}, \mathbf{w}^{N}$ и $\kappa$ могут быть неоднородны, т.е. зависеть некоторым образом от номера вершины, при этом процедура построения операторов $\mathbf{J}(A, B)$ и $J_{f}(A, B)$ никак не изменится. В этом (неоднородном) случае $\mathbf{J}$ может соответствовать или неоднородной модели Замолодчикова-Бажанова-Бакстера, или некоторой сушественной модификации этой модели (простейшей из модификаций является $N$-цветная модель на шахматной кубической решетке, основанная на модифицированном уравнении тетраэдров, см. [9]).

Помимо трансфер-матриц типа слой-слой кубической решетки, можно рассматривать трансфер-матрицы других типов, отвечающие другим сечениям кубической решетки. Или же можно рассматривать трансфер-матрицы не кубических, а, скажем, призматических трехмерных решеток (простейшей из неквадратных решеток является решетка Кагоме). В любом случае, если задана квантовая трансфер-матрица, определяюшая произвольную вспомогательную двумерную решетку с периодическими граничными условиями, то по приведенным правилам строится как оператор $\mathbf{J}(A, B)$, так и оператор $J_{f}$. Эти операторы связаны соотношением (54). Заметим, что в случае неквадратной вспомогательной решетки естественного способа ввести обозримьй $L$-оператор и развить эквивалентную технику квантового метода обратной задачи рассеяния не существует.

Благодарности. Автор хотел бы выразить искреннюю признательность Л. Д. Фаддееву, А.Г. Изергину , Г. П. Пронько, А. Г. Пронько и Ю.Г. Строганову за проявленньй к работе интерес. Особенная благодарность Игорю Корепанову и Ринату Кашаеву за исключительно полезные обсуждения в процессе получения результатов. 
Эта работа частично поддержана Российским фондом фундаментальных исследований, проект № 98-01-00070.

\section{Список литературы}

[1] A. B. Zamolodchikov. Commun. Math. Phys. 1981. V. 79. P. 489-505.

[2] V. V. Bazhanov, R. J. Baxter. J. Stat. Phys. 1992. V. 69. P. 453-485.

[3] V. V. Bazhanov, R. J. Baxter. J. Stat. Phys. 1993. V. 71. P. 839-864.

[4] R. J. Baxter. Physica D. 1986. V. 18. P. 321-347.

[5] V. V. Mangazeev, S. M. Sergeev, Yu. G. Stroganov. J. Stat. Phys. 1996. V. 82. № 1/2.

[6] S. M. Sergeev. On a two dimensional system associated with the complex of the solutions of the tetrahedron equation. solv-int/9709013; Phys. Lett. A. 1999. V. 253. № 3-4. P. 145-150; J. Phys. A. 1999. V. 32. P. 5693-5714; Solitons in a $3 d$ integrable model. solv-int/9904013; J. Nonlinear Math. Phys. 2000. V. 7. P. 1-17; C. М. Сергеев. ТМФ. 1999. T. 118. № 3. C. $479-487$.

[7] В. В. Бажсанов, Ю. Г. Строганов. ТМФ. 1982. Т. 52. № 1. С. 105-113.

[8] V.V. Bazhanov, R. M. Kashaev, V. V. Mangazeev, Yu. G. Stroganov. Commun. Math. Phys. 1991. V. 138. P. 393-408.

[9] V.V. Mangazeev, S. M. Sergeev, Yu. G. Stroganov. Int. J. Mod. Phys. A. 1994. V. 9. P. 5517-5530. 